\title{
Dynamic Modeling and Simulation of a Rotating Single Link Flexible Robotic Manipulator Subject to Quick Stops
}

\author{
Mihai Dupac* - Siamak Noroozi \\ Bournemouth University, School of Design, Engineering and Computing, U.K.
}

Single link robotic manipulators are extensively used in industry and research operations. The main design requirement of such manipulators is to minimize link dynamic deflection and its active end vibrations, and obtain high position accuracy during its high-speed motion. To achieve these requirements, accurate mathematical modeling and simulation of the initial design, to increase system stability and precision and to obtain very small amplitudes of vibration, should be considered.

In this paper the modeling of such a robotic arm with a rigid guide and a flexible extensible link subject to quick stops after each complete revolution is considered and its dynamical behavior analyzed. The extensible link that rotates with constant angular velocity has one end constrained to a predefined trajectory. The constrained trajectory allows trajectory control and obstacle avoidance for the active end of the robotic arm. The dynamic evolution of the system is investigated and the flexural response of the flexible link analyzed under the combined effect of clearance and flexibility.

Keywords: robotic arm, flexible manipulator, extensible mechanism, clearance, dynamics

\section{INTRODUCTION}

The modeling and simulation of single link manipulators have received great consideration in current years in order to improve productivity and reduce production costs. Due to the field's importance and applicability, such as to nuclear maintenance [1] and space applications [2], single link dynamics and control is considered to be a challenging research problem.

Since these manipulators contain interconnected rigid and flexible parts, their dynamic response is affected by the deformation and joint clearanceof the parts, which results in a high level of vibration and a low ability to perform accurate and safe operations. To better understand and improve their mechanical response, accurate mathematical modeling and simulation of such systems discretized using finite elements or lumped mass methods have been considered by researchers worldwide.

The vibration control of an elastic link and some active methods to eliminate its vibration was discussed in [3]. Dynamic deflection of a flexible link and its active end vibrations have been approached by Moulin and Bayo [4]. A nonlinear feedback controller for a single-link flexible manipulator derived using Lagrange approach was considered in [5]. A classical finite element approach Bricout et al. [6] was considered to study flexible manipulators. The study of interconnected rigid and flexible links using finite elements has been discussed in [7]. Dynamic finite elements models of manipulators with a flexible links was analyzed by Tokhi et al. [8], and some control strategies for damping vibration using shaping techniques have been approached in [9].

A dynamical analysis of a rotating single link manipulator was considered in [10]. Kinematic redundant manipulators with link flexibility and minimum deformation of the active end have been discussed in [11]. Lumped mass models to simulate trajectory tracking of the active end of a single-link flexible manipulator have been considered by Zhu et al. [12] and for two links manipulators in [13] and [14]. Dynamics and control of flexible-joint and dualarm robots and extensible members can be found in the papers given by [15] and [16]. The control of the vibrations of a flexible linkage mechanism and the impact effects have been discussed in [17]. The linear control a rotating flexible link of variable length undergoing periodic impacts was studied in [18].

The modeling of a translating flexible link with a prismatic joint and rotational motion and the study of its dynamical behavior have been considered in [19] and [20]. A dynamic finite element modeling of a translating and rotating flexible link was considered in [21]. The kinematic and dynamic simulation, impulsive/contact dynamics and stability of flexible mechanical systems have been presented and discussed in [22] to [24]. The dynamic analysis of some planar mechanisms with slider joints and clearance was considered in [25] and [26] and with lumped masses and impact in [27] and [28]. Mechanical systems with clearance and different types of impact/contact force models have been analyzed in [29] to [31]. A contact force model with hysteresis damping was considered in [32], and with a compliant contact in [33]. 
In this paper the modeling and simulation of an extensible robotic arm with a rigid crank and a constrained flexible link subject to quick stops is considered. The flexible link, which rotates with constant angular velocity in a horizontal plane, has one end constrained to a predefined trajectory. The constrained trajectory allows trajectory control and obstacle avoidance of the end-effector of the robotic arm. The simulations performed on a circular/circularelliptic constrained trajectory, involves quick stops after each two complete revolutions, in order to explore manipulator behavior under real operational conditions. A clearance vs. a non-clearance model of the extensible flexible arm was considered in order to analyze the effect of clearance on the dynamic behavior of the system.

\section{SINGLE LINK MANIPULATOR SYSTEM MODEL}

The flexible link PS of the single link manipulator in Fig. 1 is discretised as shown in Fig. 2 using $n$ successive equal rods $N_{i} N_{i+1}$ (where $N_{1}=P$ and $N_{n}$ $=S$ ) connected with torsional springs. The flexible link, which can slide inside the rigid guide $O D$, is represented using a fixed reference frame $O^{\prime} x y$ with the origin at $O^{\prime}$.

Each one of the successive equal rods has mass $m_{i}=m$, length $l=L / n$, and moment of inertia $J$. Each one of the springs used to model link flexibility [34] and [35] has the stiffness $k=E J / d_{P S}$ where $E$ is the Young modulus and $d_{P S}=\sum_{i=1}^{n} d_{N_{i} N_{i+1}}=L$ is the length of the flexible link, $d_{O D}$ is the length of the rigid crank and $d_{O P}$ is the distance between the end $\mathrm{O}$ of the guide and the constrained trajectory of the system.

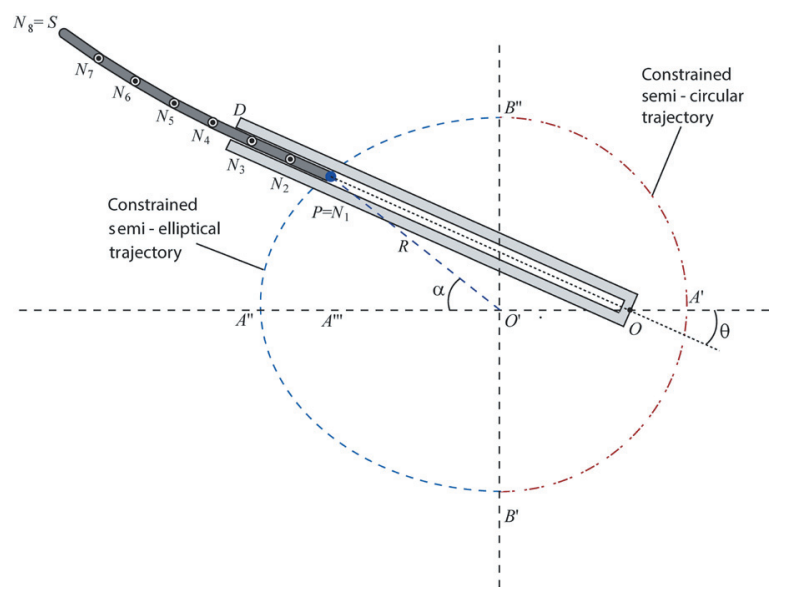

Fig. 1. Single link manipulator with a constrained flexible link and rigid support
The constrained trajectory that allows obstacle avoidance by the active end of the robotic arm is, in this case, an elliptic trajectory. The elliptical trajectory having its origin at $O^{\prime}$ has a transverse diameter of length $d_{A^{\prime} A^{\prime \prime}}$ and a conjugate diameter of length $d_{B^{\prime} B^{\prime \prime}}$. The link $P S$ is constrained to the circular/circularelliptic trajectory by a slot-joint.

The quick stop on the circular/circular-elliptic constrained trajectory takes place on the semielliptic trajectory at location $A^{\prime \prime}$ after every two complete revolutions, that is, a quarter-elliptic $A^{\prime \prime} B^{\prime}$ trajectory followed by a complete circular $B^{\prime} A^{\prime} B^{\prime \prime} A^{\prime \prime \prime} B^{\prime}$ trajectory, followed by a semi-circular $B^{\prime} A^{\prime} B^{\prime \prime}$ trajectory and finally followed by a quarter-elliptic $B^{\prime \prime} A$ " trajectory, and so on. The angle between two successive links $N_{i} N_{i+1}$ and $N_{i+1} N_{i+2}$ is denoted by $\theta_{i+1}$ for any $i=\overline{1, n-1}$, while the angle between the link $N_{i} N_{i+1}$ and the horizontal direction is denoted by $\Theta_{i}$.

The angle between the guide and the horizontal direction denoted by $\theta$, is always equal to the angle $\theta_{1}$ if there is no clearance between the guide and the flexible link. The motor torque, which acts on the end $O$ of the rigid guide, performs quick stops after each complete revolution.

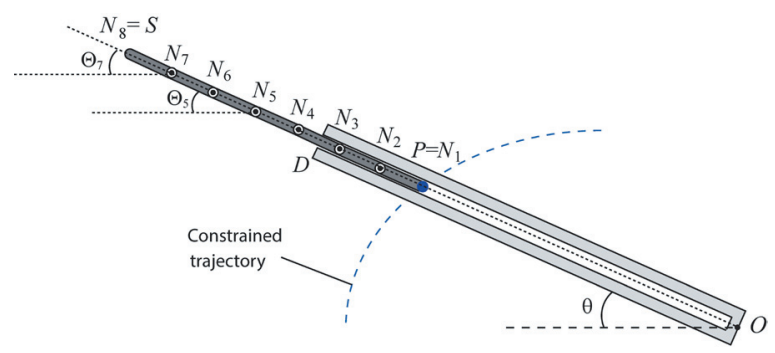

Fig. 2. Lumped mass model of the flexible link with rigid support (guide)

\section{DYNAMIC MODEL OF THE SINGLE LINK MANIPULATOR}

The dynamic model for the constrained flexible link can be expressed based on the position, velocity and acceleration vector of the centre of the mass $C_{i}$ of each rigid $\operatorname{rod} N_{i} N_{i+1}, i=\overline{1, n}$.

The position vector of the mass centre $C_{i}$ of each rigid $\operatorname{rod} N_{i} N_{i+1}$ is given by $\mathbf{r}_{C_{i}}=x_{C_{i}} \mathbf{i}+y_{C_{i}} \mathbf{j}$, where $i$ and $j$ are the unit vectors of the fixed reference frame $O ' x y$. The horizontal and the vertical coordinates $x_{C_{i}}$ and $y_{C_{i}}$ can be expressed as:

$$
\begin{aligned}
{\left[\begin{array}{l}
x_{C_{i}}= \\
y_{C_{i}}=
\end{array}\right]=} & d_{O N_{1}}\left[\begin{array}{c}
\cos \theta \\
\sin \theta
\end{array}\right]+\sum_{j=1}^{i} d_{N_{j} N_{j+1}}\left[\begin{array}{c}
\cos \Theta_{j} \\
\sin \Theta_{j}
\end{array}\right]- \\
& -\frac{1}{2} d_{N_{i} N_{i+1}}\left[\begin{array}{l}
\cos \Theta_{i} \\
\sin \Theta_{i}
\end{array}\right],
\end{aligned}
$$


where the distance $d_{O N_{1}}$ (computed as in [36]) represents the variable distance between the centre of rotation of the guide denoted by $O$ and the constrained trajectory, that is, the considered circular/circularelliptic trajectory. It can be observed that if first $k$ connecting rods $N_{j} N_{j+1}, j=\overline{1, k}$ are inside the driver $O D$, that is $d_{O N_{1}}+\sum_{j=1}^{k} d_{N_{j} N_{j+1}} \leq d_{O D}$, then $\Theta_{j}=\theta$, for all $j=\overline{1, k}$.

The velocity vector of the mass centre $C_{i}$ of each rigid rods $N_{i} N_{i+1}$ is the derivative with respect to time of the position vector $\mathbf{r}_{C_{i}}$ given by $\mathbf{v}_{C_{i}}=\dot{\mathbf{r}}_{C_{i}}=\dot{x}_{C_{i}} \mathbf{i}+\dot{y}_{C_{i}} \mathbf{j}$ where:

$$
\begin{aligned}
& {\left[\begin{array}{l}
v_{x_{C_{i}}} \\
v_{y_{C_{i}}}
\end{array}\right]=\left[\begin{array}{l}
\dot{x}_{C_{i}} \\
\dot{y}_{C_{i}}
\end{array}\right]=\dot{d}_{O N_{1}}\left[\begin{array}{c}
\cos \theta \\
\sin \theta
\end{array}\right]+d_{O N_{1}} \dot{\theta}\left[\begin{array}{c}
-\sin \theta \\
\cos \theta
\end{array}\right]+} \\
& +\sum_{j=1}^{i} d_{N_{j} N_{j+1}} \dot{\Theta}_{j}\left[\begin{array}{c}
-\sin \Theta_{j} \\
\cos \Theta_{j}
\end{array}\right]+\frac{1}{2} d_{N_{i} N_{i+1}} \dot{\Theta}_{i}\left[\begin{array}{c}
\sin \Theta_{i} \\
-\cos \Theta_{i}
\end{array}\right] .
\end{aligned}
$$

The acceleration vector of the mass centre $C_{i}$ of each rigid rods $N_{i} N_{i+1}$ is the double derivative with respect to time of the position vector $\mathbf{r}_{C_{i}}$ given by $\mathbf{a}_{C_{i}}=\ddot{\mathbf{r}}_{C_{i}}=\ddot{x}_{C_{i}} \mathbf{i}+\ddot{y}_{C_{i}} \mathbf{j}$ where:

$$
\begin{aligned}
& {\left[\begin{array}{l}
a_{x_{C_{i}}} \\
a_{y_{C_{i}}}
\end{array}\right]=\ddot{d}_{O N_{1}}\left[\begin{array}{c}
\cos \theta \\
\sin \theta
\end{array}\right]+2 \dot{d}_{O N_{1}} \dot{\theta}\left[\begin{array}{c}
-\sin \theta \\
\cos \theta
\end{array}\right]+} \\
& +d_{O N_{1}}\left(\ddot{\theta}\left[\begin{array}{c}
-\sin \theta \\
\cos \theta
\end{array}\right]-\dot{\theta}^{2}\left[\begin{array}{c}
\cos \theta \\
\sin \theta
\end{array}\right]\right)- \\
& -\sum_{j=1}^{i} d_{N_{j} N_{j+1}}\left(\dot{\Theta}_{j}^{2}\left[\begin{array}{c}
\cos \Theta_{j} \\
\sin \Theta_{j}
\end{array}\right]+\ddot{\Theta}_{j}\left[\begin{array}{c}
-\sin \Theta_{j} \\
\cos \Theta_{j}
\end{array}\right]\right)+ \\
& +\frac{1}{2} d_{N_{i} N_{i+1}}\left(\ddot{\Theta}_{i}\left[\begin{array}{c}
\sin \Theta_{i} \\
-\cos \Theta_{i}
\end{array}\right]+\dot{\Theta}_{i}^{2}\left[\begin{array}{c}
\cos \Theta_{i} \\
\sin \Theta_{i}
\end{array}\right]\right) .
\end{aligned}
$$

\subsection{Single Link Manipulator with No Clearance}

For the model with no clearance the flexible link translates parallel to its support and exhibits a continuous contact with the guide. One can write the Lagrange differential equation of motion with no impact as:

$$
\frac{d}{d t}\left(\frac{\partial T}{\partial \dot{q}_{i}}\right)-\frac{\partial T}{\partial q_{i}}=Q_{i}
$$

where $T$ is the total kinetic energy of the system, $T_{i}$ is the kinetic energy of each $i^{\text {th }}$ link, $q_{i}=\theta_{i}$ are the generalized coordinates, $Q_{i}$ are the generalized forces and the subscript $i$ represents the number of the generalized forces/coordinates.

The total kinetic energy can be expressed as in [27] by:

$$
T=\sum_{i=1}^{n} T_{i}=\frac{1}{2} \sum_{i=1}^{n}\left(m_{i} \mathbf{v}_{C_{i}}^{2}+I_{C_{i}} \omega_{i}^{2}\right)
$$

where the angular velocities $\boldsymbol{\omega}_{i}$ can be expressed as $\boldsymbol{\omega}_{i}=\Theta_{i} \mathbf{k}$ for all $i=\overline{1, n}$. The model described in [27] was used to describe the generalized forces acting on each link.

\subsection{Single Link Manipulator with Clearance}

The same clearance model considered in [34], where the flexible link of the manipulator model can translate and rotate about its support, was used for this study. Due to the clearance model, the flexible link may impact the guide as shown in Fig. 3.

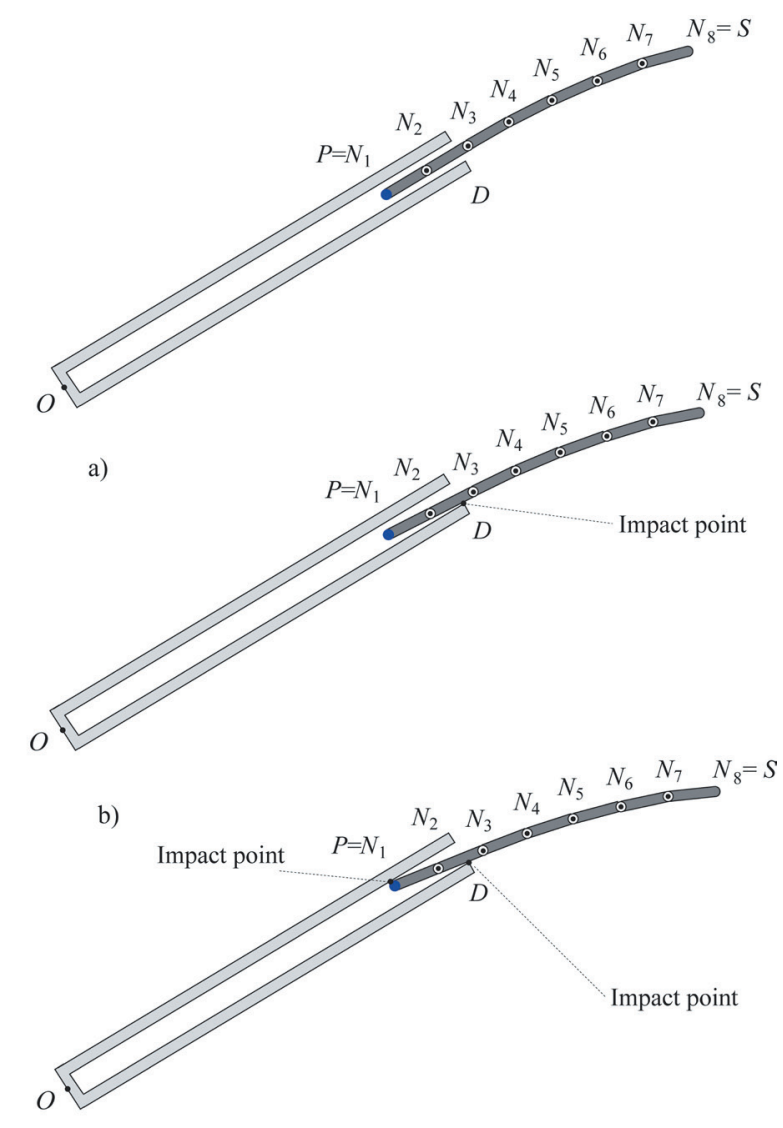

c)

Fig. 3. Constrained flexible extensible link with rigid support and clearance model; a) no impact, b) impact on one point, and c) impact on two points 
The impact model between the flexible link and the guide is shown in Fig. 3. Possible impact cases between the flexible link and the guide are: a) no impact, b) impact on a single point, and c) impact on two points. Since multiple impacts at the same time instant can be statistically excluded no such case was considered in this study. The discontinuous model considered here [27] and [18] assumes instantaneous impact and no change in the system configuration during contact, that is, the integration of equations of motion is halted at the time of impact, a momentum balance is performed to calculate the post impact velocities of the system components, and energy dissipation is quantified using the restitution coefficient. Other types of impact/contact force models have been discussed in [29], [30] and [31]. A hysteresis contact force model was discussed in [32], and a compliant contact in [33].

Using the discontinuous model [27] and [18] the impact differential equation of motion can be written as:

$$
\left(\frac{\partial T}{\partial \dot{u}_{j}}\right)_{t_{s}}-\left(\frac{\partial T}{\partial \dot{u}_{j}}\right)_{t_{a}}=P_{j},
$$

where $\frac{\partial T}{\partial \dot{u}_{j}}$ are the generalized momenta and $T$ is the total kinetic energy of the system, $P_{j}$ are the generalized impulses associated with the coordinate $u_{j}$, and $t_{a}$ and $t_{s}$ represents the time of approach and separation. Considering:

$$
\int_{t_{a}}^{t_{s}} \mathbf{R} d t=R_{x} \mathbf{i}+R_{y} \mathbf{j}
$$

the force exerted during the impact of the flexible link with the guide. One can express the generalized impulses by:

$$
P_{j}=\sum \frac{\partial \mathbf{v}_{L}}{\partial q_{j}} \int_{t_{a}}^{t_{s}} \mathbf{R} d t
$$

where $\mathbf{v}_{L}=\mathbf{v}_{G}+\boldsymbol{\omega} \times \mathbf{r}_{N}$ is the velocity of the impact point when the guide comes in contact with the flexible link. The velocity of approach and separation on the flexible link and guide can be expressed as:

$$
\left\{\begin{array}{l}
\mathbf{v}_{a}=\left.\mathbf{v}_{L}\right|_{t_{a}}-\left.\mathbf{v}_{G}\right|_{t_{a}}, \\
\mathbf{v}_{s}=\left.\mathbf{v}_{L}\right|_{t_{s}}-\left.\mathbf{v}_{G}\right|_{t_{s}}
\end{array}\right.
$$

where $\left.\mathbf{v}_{L}\right|_{t_{a}}$ and $\left.\mathbf{v}_{G}\right|_{t_{a}},\left.\mathbf{v}_{L}\right|_{t_{s}}$ and $\left.\mathbf{v}_{G}\right|_{t_{s}}$ are the flexible link velocity and guide velocity at time $t_{a}$ and $t_{s}$ before and after the impact. Using Newton's formulation for the definition of the coefficient of restitution e and Eq. (9), one can write:

$$
-e \mathbf{v}_{a}=\mathbf{v}_{s} .
$$

\section{SIMULATION AND RESULTS}

In this section results from the computer simulations are presented for the following manipulator dimensions and material properties: guide length and clearance of $0.09 \mathrm{~m}$ and $3 \times 10^{-3} \mathrm{~m}$ respectively, a constrained flexible link of $0.07 \mathrm{~m}$, density of 7850 $\mathrm{kgm}^{-3}$, Young's modulus of $2 \cdot 1011 \mathrm{~Pa}$, and Poisson ratio of 0.3 . The rotating end of the guide is located on the $O x$ axis at $0.02 \mathrm{~m}$ from the origin of the Cartesian reference frame. The constrained trajectory, that is, a semi-circular semi-elliptic trajectory is described as follows. The principal axes of the semi-elliptic trajectory, with the origin located on the $O x$ axis at
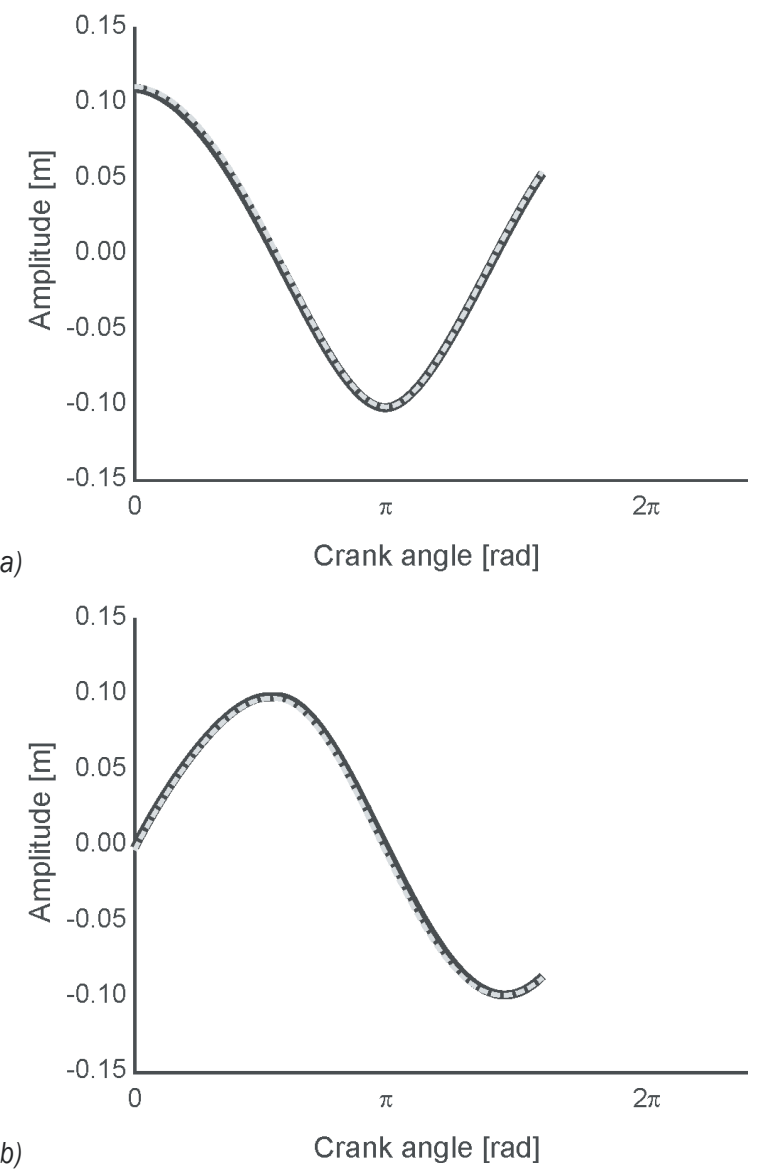

Fig. 4. Dynamic behaviour of the extensible flexible link with a quick stop and angular velocity $\omega_{1}$; a) $0 x$, and b) $0 y$, trajectories with (black/solid) and without (lighter/dashed) clearance for the active end of the single link manipulator 
$0.01 \mathrm{~m}$ from the origin of the Cartesian reference frame, are described by a transverse diameter of 0.08 $\mathrm{m}$, a conjugate diameter of $0.06 \mathrm{~m}$. The semi-circle with the origin located on the $O x$ axis at $0.01 \mathrm{~m}$ from the origin of the Cartesian reference has a radius of $0.03 \mathrm{~m}$.

The effect of friction has been neglected in this study, and the contacts/impacts of the flexible link with the crank are considered to be frictionless, that is, the friction coefficient between the parts was neglected. The dynamic behaviour of the constrained single link manipulator rotating with the angular velocity $\omega_{1}$, with no clearance and quick stop is shown in Fig. 4a, that is, the $O x$ and $O y$ trajectories of the active end of the flexible link are plotted vs. the crank angle.

From Fig. 4a one can observe the perturbation the system due to the quick stop, as well as the time frame in which the system damped the perturbation. Since a perturbation of the trajectory is an important factor affecting system dynamics, the behaviour of the extensible flexible link for a clearance vs. a nonclearance model have been considered.

The dynamic behaviour of the constrained single link manipulator, with clearance and quick stops is shown in Fig. 4b, that is, the $O x$ and $O y$ trajectories of the active end of the flexible link plotted vs. the crank angle. It was observed that the quick stop added more excitation to the end of the flexible link while impacts due to clearance act against the quick stop excitation and about the beam as a whole as shown in Figs. 4a and $b$.

Since an important design requirement of a rotating manipulator is the minimisation of the link dynamic deflection during high-speed motion, the effect of a higher angular velocity for the same clearance vs. a non-clearance model was also considered. The dynamic behaviour of the constrained single link manipulator with no clearance and angular velocity $\omega_{1}$ is shown in Fig. 5a.

A higher perturbation of the system trajectory can be observed for the angular velocity $\omega_{2}$ in Fig. 5a vs. the angular velocity $\omega_{1}$ in Fig. 4a. The dynamical behaviour of the single link manipulator with clearance and angular velocity $\omega_{2}$ is shown in Fig. 5b. A similar behaviour, that is, a higher trajectory perturbation, can be observed for the angular velocity $\omega_{2}$ in Fig. 5 b vs. the angular velocity $\omega_{1}$ in Fig. 4b. It was observed that more excitation is added to the system due to the quick stop against the impacts due to clearance acting as shown in Figs. 5a and 5b. It was also observed that the quick stops performed against the single link manipulator amplify the dynamic forces and flexible link vibration amplitude (against the decrease in the flexible link natural frequency with length increase) as shown in Figs. 5 and 6.
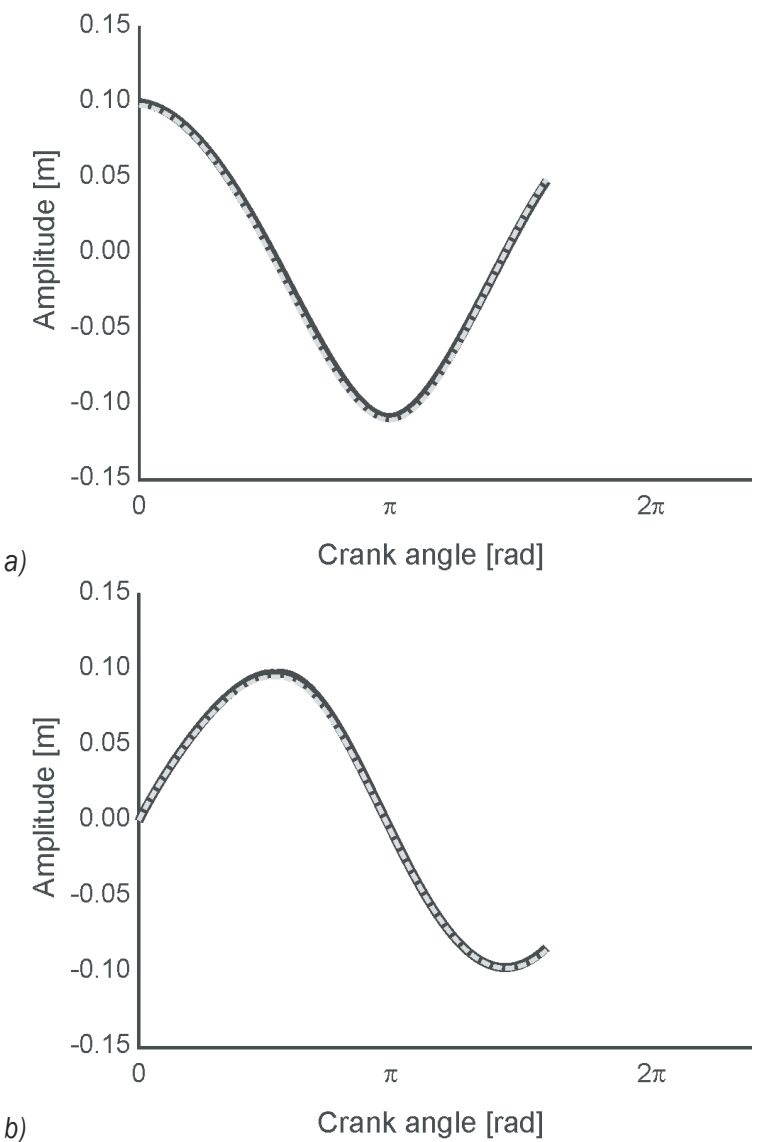

Fig. 5. Dynamic behaviour of the extensible flexible link with a quick stop and angular velocity $\omega_{2}$; a) $0 x$, and b) Oy, trajectories with(black/solid) and without (lighter/dashed) clearance for the active end of the single link manipulator

However, the decrease in the flexible link length due to the motion along the constrained circularelliptic trajectory increases the stiffness of the link, which was confirmed in a relatively similar study [37] regarding the behaviour of a flexible robot manipulator with a rotating-prismatic joint. Only the first mode of vibration have been observed for the vibrating flexible link with no clearance, while for the clearance model, the flexible link exhibits both the first and the second mode of vibration.

The displacement of the active end of the flexible manipulator with clearance along the active trajectory (Figs. 6a and b), immediately after the quick stop and for both angular velocities $\omega_{1}$ and $\omega_{2}$, that is, the interactions of each vibration mode, is shown in Figs. $7 \mathrm{a}$ and $\mathrm{b}$, respectively. 


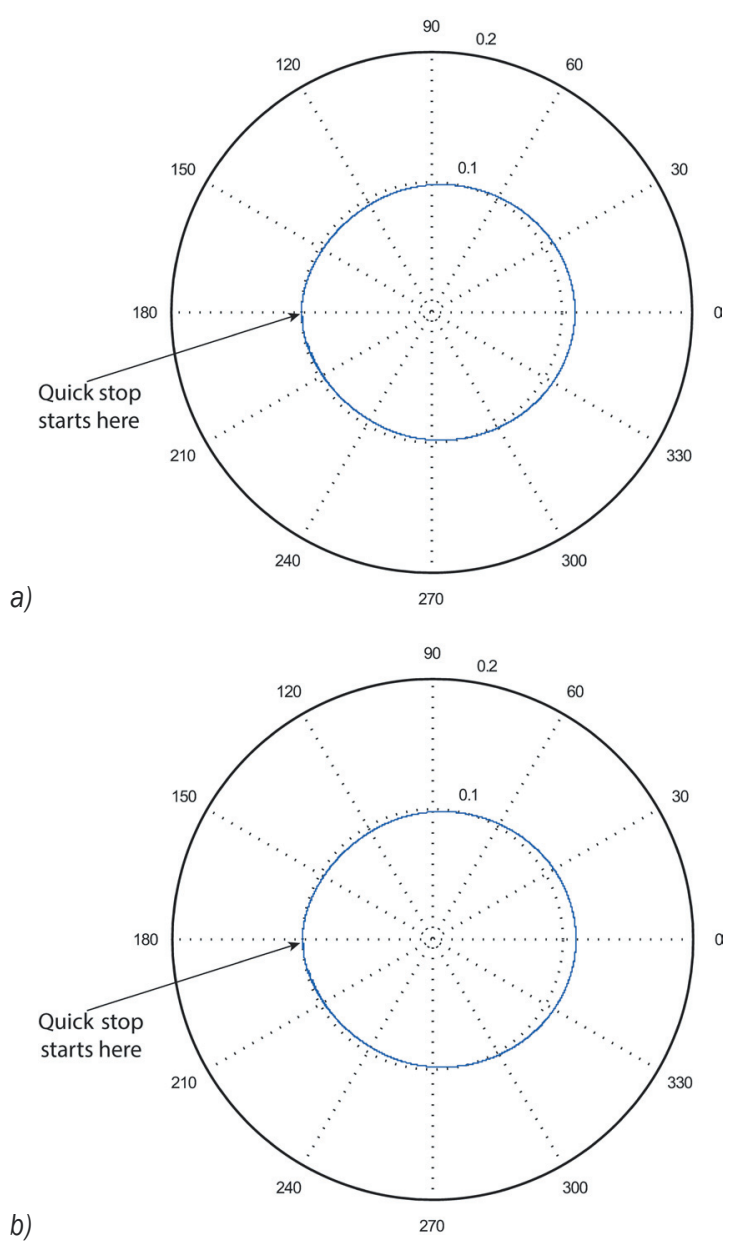

Fig. 6. Trajectories of the active end flexible manipulator with clearance for; a) angular velocity $\omega_{1}$, and b) angular velocity $\omega_{2}$

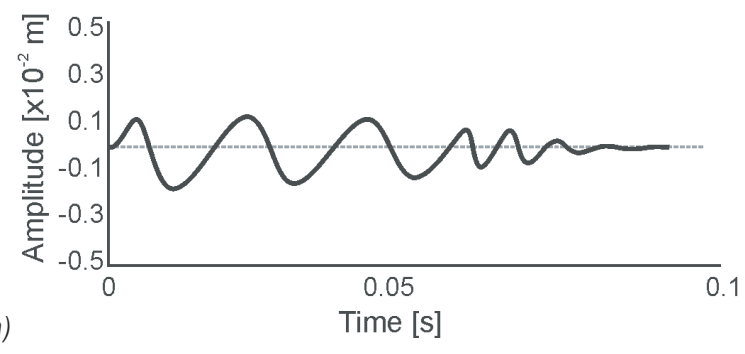

a)

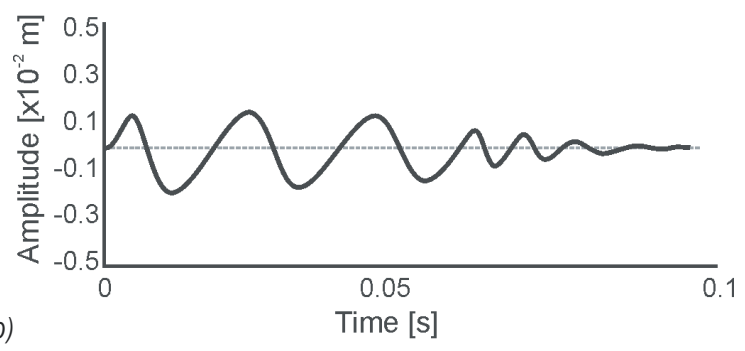

Fig. 7. Dynamic behaviour of the active end of the flexible manipulator with clearance along the active trajectory immediately after the quick stop, a) active vibrations for angular velocity $\omega_{1}$, and b) active vibrations for angular velocity $\omega_{2}$
It can be seen that the amplitude of vibration suddenly increases immediately after the quick stops, due to the increase in the dynamic force. The two types of behaviour (Figs. 7a and b respectively) are nearly identical and the small differences are mainly due to the different angular velocities, which slightly change the dynamic force acting on the flexible link.

For the mechanism simulations presented here a nine rigid rods approximation has been used, that is, nine rigid links connected with torsional springs have been used to simulate the elastic rod.
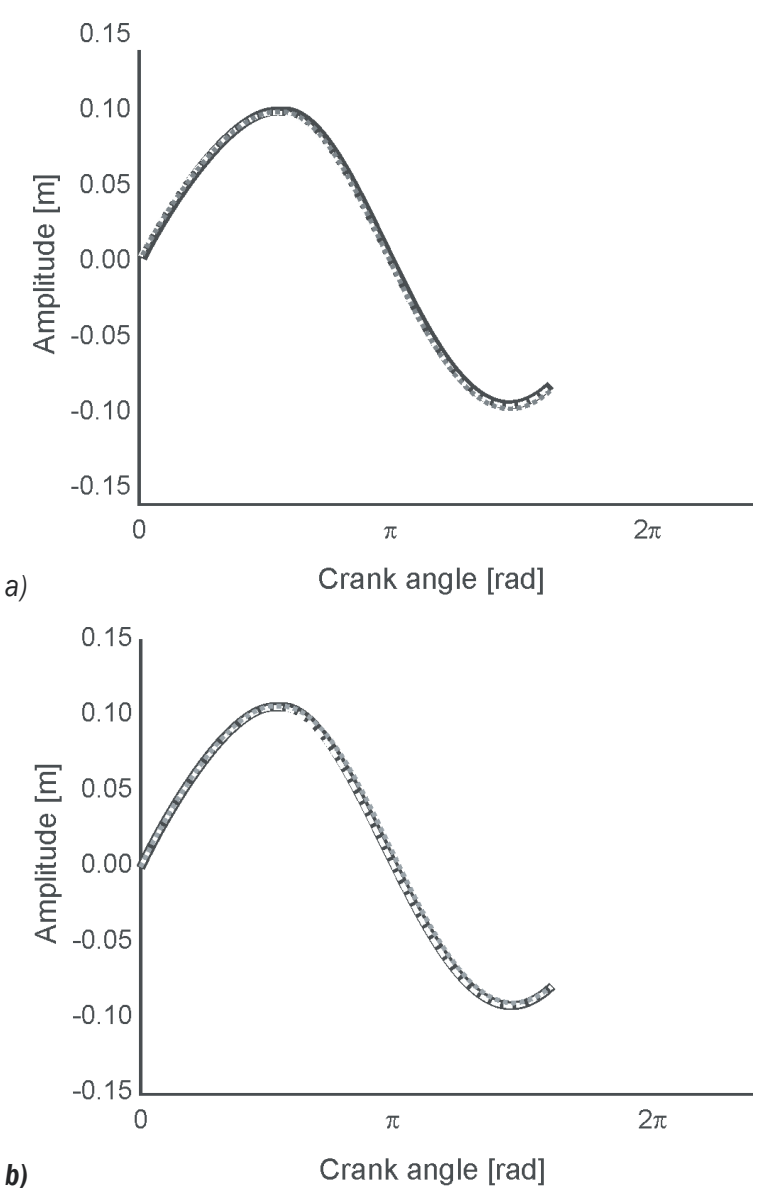

Fig. 8. Dynamic behaviour of the active end flexible manipulator with clearance along the active trajectory immediately after the quick stop, Oy trajectories; a) without; and b) with clearance for 2 (gray/dotted), 4 (black) and 9 (white/dashed) links simulation

To validate the obtained results, the effect of the number of elements on the dynamic response of the system was considered. Simulations involving 2 and 4 roads performed and compared to the nine rod simulation present visible differences between the obtained trajectories as shown in Fig 8. Simulations involving 8 and 11 rods have been performed too and no perceptible difference was found with respect to 
the dynamic behaviour of the 9 rod simulation. Thus $n=9$ was considered adequate to accurately describe the mechanism dynamics.

One can conclude that the time evolution of the system is mainly affected by the quick stops and angular velocity, factors which accentuate trajectory divergence and affect system stability.

\section{CONCLUSIONS}

In this paper the modelling and dynamic response of a constrained single link flexible rotating manipulator subject to quick stops was investigated. One end of the flexible manipulator was constrained to a predefined trajectory for possible trajectory control and obstacle avoidance. The effect of link flexibility, axial shortening and clearance has been considered in the analysis of the rotating flexible manipulator. It was observed that the time evolution of the system was mainly affected by the quick stops and angular velocity. At the time of the quick stop (which represents the main impacts of the system) the flexible link makes successive shocks, which increase the amplitude of vibration. Further experimental tests will be performed in order to validate and generalize the simulations reported in this study.

\section{REFERENCES}

[1] Mitiguy, P., Banerjee, A.K., (2000). Determination of spring constants for modelling flexible beams. Working Model Technical Paper.

[2] Miyabe, T., Konno, A., Uchiyama, M. (2003). Automated object capturing with a two arm flexible manipulator. Proceedings of the IEEE International Conference on Robotics and Automation, vol. 2, p. 2529-2534, DOI:10.1109/ROBOT.2003.1241973.

[3] Ankarali, A., Diken, H. (1997). Vibration control of an elastic manipulator link. Journal of Sound and Vibration, vol. 204, no. 1, p. 162-170, DOI:10.1006/ jsvi.1996.0897.

[4] Moulin, H., Bayo, E. (1991). On the accuracy of endpoint trajectory tracking for flexible arms by noncausal inverse dynamic solutions. ASME Journal of Dynamic Systems, Measurement, and Control, vol. 113, no. 2, p. 320-324, DOI:10.1115/1.2896384.

[5] Ge, S.S., Lee, T.H., Zhu, G. (1998). A nonlinear feedback controller for a single-link flexible manipulator based on finite element model. Journal of Field Robotics, vol. 14 , no. 3 , p. 165-178, DOI:10.1002/(SICI)10974563(199703)14:3<165::AID-ROB2>3.0.CO;2-P.

[6] Bricout, J.N., Debus, J.C., Micheau, P. (1990). A finite element model for the dynamics of flexible manipulator. Mechanism and Machine Theory, vol. 25, no.1, p. 119128, DOI: 10.1016/0094-114X(90)90111-V.
[7] Nagarajan, S., Turcic, D.A. (1990). Lagrangian formulation of the equations of motion for the elastic mechanisms with mutual dependence between rigid body and elastic motions, Part 1: Element level equations ASME Journal of Dynamic Systems, Measurement, and Control, vol. 112, no. 2 p. 203-214, DOI:10.1115/1.2896127.

[8] Tokhi, M.O., Mohamed, Z., Shaheed, M.H. (2001). Dynamic characterisation of a flexible manipulator system. Robotica, vol. 19, no. 5, p. 571-580, DOI:10.1017/S0263574700003209.

[9] Mohamed, Z., Tokhi, M.O. (2004). Command shaping techniques for vibration control of a flexible robot manipulator. Mechatronics, vol. 14, no. 1, p. 69-90, DOI:10.1016/S0957-4158(03)00013-8.

[10] Chung, J., Yoo, H.H. (2002). Dynamic analysis of a rotating cantilever beam by using the finite element method. Journal of Sound and Vibration, vol. 249, no.1, p. 147-164, DOI:10.1006/jsvi.2001.3856.

[11] Yue, S.G. (1998). Redundant robot manipulators with joint and link flexibility I: dynamic motion planning for minimum end-effector deformation. Mechanism and Machine Theory, vol. 33, no. 1-2, p. 103-113, DOI:10.1016/S0094-114X(97)00028-1.

[12] Zhu, G., Ge, S.S., Lee, T.H. (1999). Simulation studies of tip tracking control of a single-link flexible robot based on a lumped model. Robotica, vol. 17, p. 71-78, DOI:10.1017/S0263574799000971.

[13] Benosman, M., Vey, G.L., Lanari, L., De Luca, A. (2004). Rest-to-rest motion for planar multi link flexible manipulator through backward recursion. ASME Journal of Dynamic Systems Measurement and Control, vol. 126, no. 1, p. 115-123, DOI:10.1115/1.1649976.

[14] Green, A., Sasiadek, J.Z. (2004). Dynamics and trajectory tracking control of a two-link robot manipulator. Journal of Vibration and Control, vol. 10, p. 1415-1440, DOI:10.1177/1077546304042058.

[15] Liu, S., Wu, L., Lu, Z. (2007). Impact dynamics and control of a flexible dual-arm space robot capturing an object. Applied Mathematics and Computation, vol. 185, no. 2, p. 1149-1159, DOI:10.1016/j. amc.2006.07.035.

[16] Zhang, D.-G., Angeles, J. (2005). Impact dynamics of flexible-joint robots. Computers \& Structures, vol. 83, no. 1, p. 25-33, DOI:10.1016/j.compstruc.2004.08.006

[17] Jin, C., Fan, L., Qiu, Y. (2004). The vibration control of a flexible linkage mechanism with impact. Communications in Nonlinear Science and Numerical Simulation, vol. 9, no. 4, p. 459-469, DOI:10.1016/ S1007-5704(02)00134-X.

[18] Marghitu, D.B., Sinha, S.C., Diaconescu, C. (1999). Control of a parametrically excited flexible beam undergoing rotations and impacts. Multibody System Dynamics, vol. 3, no. 1, p. 47-63, DOI:10.1023/A:1009716921661.

[19] Basher, A.M.H. (2000). Dynamic behavior of a translating flexible beam with a prismatic joint. 
Proceedings of the IEEE Southeastcon, Nashville, p. 31-38.

[20] Yuh, J., Young, T., Baek, Y.S. (1989). Modeling of Flexible Link having a Prismatic Joint in Robot Mechanism - Experimental Verification. IEEE International Conference on Robotics and Automation, Proceedings, Scottsdale, vol. 2, p. 722-727, DOI:10.1109/ROBOT.1989.100070.

[21] Al-Bedoor, B.O., Khulief, Y.A. (1996). Finite element dynamic modeling of a translating and rotating flexible link. Computer Methods in Applyed Mechanics and Engineering, vol. 131, no. 1-2, 173-189, DOI:10.1016/0045-7825(95)00968-X.

[22] Beale, D., Lee, S.W., Boghiu, D. (1998). An analytical study of fuzzy control of a flexible rod mechanism. Journal of Sound and Vibration, vol. 210, no.1, p. 3752, DOI:10.1006/jsvi.1997.1266.

[23] Garcia, J., Bayo, J.E. (1994). Kinematic and Dynamic Simulation of Multibody Systems, Springer, New York, DOI:10.1007/978-1-4612-2600-0.

[24] Kvecses, J., Cleghorn, W.L. (2004). Impulsive dynamics of a flexible arm: analytical and numerical solutions. Journal of Sound and Vibration, vol. 269, no. 1-2, p. 183-195, DOI:10.1016/S0022-460X(03)00068-3.

[25] Stoenescu, E.D., Marghitu, D.B. (2003). Dynamic analysis of a planar rigid-link mechanism with rotating slider joint and clearance. Journal of Sound and Vibration, vol. 266, no. 2, p. 394-404, DOI:10.1016/ S0022-460X(03)00053-1.

[26] Zhuang, F., Wang, Q. (2013). Modeling and simulation of the nonsmooth planar rigid multibody systems with frictional translational joints. Multibody System Dynamics, vol. 29, no. 4, p. 403-423, DOI:10.1007/ s11044-012-9328-5.

[27] Dupac, M., Marghitu, D.B. (2006). Nonlinear dynamics of a flexible mechanism with impact. Journal of Sound and Vibration, vol. vol. 289, no. 4-5, 952-966, DOI:10.1016/j.jsv.2005.03.002.

[28] Rubinstein, D. (1999). Dynamics of a flexible beam and a system of rigid rods, with fully inverse (onesided) boundary conditions. Computer Methods in Applied Mechanics and Engineering, vol. 175, no. 1-2, p. 87-97, DOI:10.1016/S0045-7825(98)00321-1.
[29] Brach, R.M. (1989). Rigid body collisions. Journal of Applied Mechanics, vol. 56, no. 1, p. 133-138, DOI:10.1115/1.3176033.

[30] Flores, P., Leine, R., Glocker, C. (2010). Modeling and analysis of planar rigid multibody systems with translational clearance joints based on the non-smooth dynamics approach. Multibody System Dynamics, vol. 23, no. 2, p. 165-190, DOI:10.1007/s11044-0099178-y.

[31] Flores, P., Machado, M., Silva, M.T., Martins, J.M. (2011). On the continuous contact force models for soft materials in multibody dynamics. Multibody System Dynamics, vol. 25, no. 3, p. 357-375, DOI:10.1007/ s11044-010-9237-4.

[32] Lankarani, H.M., Nikravesh, P.E. (1990). A contact force model with hysteresis damping for impact analysis of multibody systems. Journal of Mechanical Design, vol. 112, no. 3, p. 369-376, DOI:10.1115/1.2912617.

[33] Machado, M., Moreira, P., Flores, P., Lankarani, H.M. (2012). Compliant contact force models in multibody dynamics: Evolution of the Hertz contact theory. Mechanism and Machine Theory, vol. 53, p. 99-121, DOI:10.1016/j.mechmachtheory.2012.02.010.

[34] Dupac, M. (2013). Dynamic Analysis of a Constrained Flexible Extensible Link with Rigid Support and Clearance. Journal of Theoretical and Applied Mechanics, (In Press).

[35] Meggiolaro, M.A., Dubowsky, S. (2001). Improving the positioning accuracy of powerful manipulators with application in nuclear maintenance. Proceedings of the $16^{\text {th }}$ Brazilian Congress in Mechanical Engineering, Uberlandia, vol. 15, p. 210-219 from: http://meggi. usuarios.rdc.puc-rio.br/paper/C030_COBEM01_ Improving_the positioning.pdf.

[36] Dupac, M. (2013). A virtual prototype of a constrained extensible crank mechanism: Dynamic simulation and design. Proceedings of the Institution of Mechanical Engineers, Part K: Journal of Multi-body Dynamics, vol. 227, no. 3, 201-210, DOI:10.1177/1464419313479531.

[37] Kalyoncu, M. (2008). Mathematical modelling and dynamic response of a multistraight-line path tracing flexible robot manipulator with rotating-prismatic joint. Applied Mathematical Modelling, vol. 32, no. 6, p. 1087-1098, DOI:10.1016/j.apm.2007.02.032. 